\title{
A THEOREM ON CONTINUED FRACTIONS
}

\author{
by G. N. WATSON
}

(Received 15th December 1958; revised MS. received 5th January 1959)

\section{Introduction}

One of the outstanding theorems in the theory of continued fractions is the result described by $\mathrm{O}$. Perron, Die Lehre von den Kettenbrüchen (1912, 1929, 1954-7) as the transformation of Bauer and Muir (for brevity I shall call it the BM theorem); this theorem and limiting cases of it give rise to numerous extremely interesting consequences.

Recently I had occasion to use a special case of the BM theorem, and I was thereby led to study the theorem itself. It then seemed to me that the proof of the theorem was more intricate than one would wish in so fundamental a result, and I consequently set about trying to construct a proof with quite a different starting point.

The upshot was that I constructed a relation connecting two continued fractions which was apparently more general than the BM theorem in that it involved four arbitrary sequences in place of the three which occur in the BM theorem (though, as will be seen in $\$ 5$, this apparent increase of generality is spurious) ; and, what seems more important, my result possesses a certain amount of symmetry which is completely lacking in the BM theorem in its current version. It is quite easy to display the BM theorem as a special case of my theorem, and indeed to generalise the BM theorem in a minor respect (see \$4) by a slight change of notation so that my theorem differs from the BM theorem only in the possession of the additional (removable) sequence.

It should be stated that Bauer and Muir attain their result by postulating one continued fraction and then constructing a second continued fraction in which the numerators and denominators of successive convergents are derived in a simple manner from the convergents of the first, whereas my procedure is to construct step by step two continued fractions the expressions at each step being connected by symmetrical relations. This is sufficient to account for the gain in symmetry in my work, while the simplicity which I think that I have gained by my procedure is in the nature of a fortunate accident.

It seems to me to be likely that the reason why the exposition by Perron follows the work of Bauer and Muir fairly closely is that the BM theorem is adequate to deal with all the examples for which it was needed, and so there was no necessity to seek for changes in notation or for simplifications in the proof of the theorem.

After these preliminaries I embark on the construction of my theorem, without reference to the BM theorem, and, when I have established my version of the theorem, I shall proceed to consider the connexion between the two theorems. I should, however, state at the outset that it is the general form E.M.S.-L 
of my theorem which seems to me to be interesting, and accordingly I shall not be so meticulous as to discuss special cases which may arise in consequence of continued fractions becoming nugatory; my exclusion of such cases will be covered, whenever it seems advisable, by the statement that my results hold in general.

\section{The Construction of the Theorem}

Let $n$ be any given positive integer and let $\left(x_{m}\right),\left(y_{m}\right),\left(z_{m}\right)$ be three arbitrary sequences with $m$ running through the values $0,1,2, \ldots, n$. It is convenient to defer the introduction of the fourth sequence until we have made further progress. It is also convenient to regard the members of these three sequences as constants which are completely unrestricted apart from the limitations that no member of any of them may be zero and also that $x_{m} y_{m}-z_{m}$ may not be zero for $0 \leqslant m \leqslant n$.

We next consider $2 n+2$ variables, $\theta_{m}$ and $\phi_{m}$ with $m$ again running through the values $0,1,2, \ldots, n$. We make the following hypotheses about the variables : (i) $\theta_{n}$ and $\phi_{n}$ are connected by the bilinear relation

$$
\left(\theta_{n}+x_{n}\right)\left(\phi_{n}+y_{n}\right)=z_{n} ;
$$

(ii) there are $4 n$ determinate non-zero constants $a_{m}, b_{m}, c_{m}, d_{m}$ with $m$ running through the values $1,2,3, \ldots, n$ such that

$$
\theta_{m-1}=\frac{a_{m}}{b_{m}+\theta_{m}}, \quad \phi_{m-1}=\frac{c_{m}}{d_{m}+\phi_{m}},
$$

or, what amounts to the same thing,

$$
\theta_{m}=\left(a_{m} / \theta_{m-1}\right)-b_{m}, \quad \phi_{m}=\left(c_{m} / \phi_{m-1}\right)-d_{m} ;
$$

and (iii) the constants specified in (ii) are to be determined, if possible, so that the bilinear relations (which obviously exist) connecting $\theta_{m}$ with $\phi_{m}$ are to be

$$
\left(\theta_{m}+x_{m}\right)\left(\phi_{m}+y_{m}\right)=z_{m}, \quad(m=0,1,2, \ldots, n-1) .
$$

We now carry out a step-by-step procedure to determine $a_{m}, b_{m}, c_{m}, d_{m}$ as completely as we can from these hypotheses. When, for any particular value of $m$, it has been proved that

$$
\left(\theta_{m}+x_{m}\right)\left(\phi_{m}+y_{m}\right)=z_{m},
$$

it is evident that the bilinear relation connecting $\theta_{m-1}$ with $\phi_{m-1}$ is

$$
\left(\frac{a_{m}}{\theta_{m-1}}+x_{m}-b_{m}\right)\left(\frac{c_{m}}{\phi_{m-1}}+y_{m}-d_{m}\right)=z_{m} .
$$

Necessary and sufficient conditions for this relation to be equivalent to the relation

$$
\left(\theta_{m-1}+x_{m-1}\right)\left(\phi_{m-1}+y_{m-1}\right)=z_{m-1}
$$


are obviously

$$
\frac{\left(x_{m}-b_{m}\right)\left(y_{m}-d_{m}\right)-z_{m}}{1}=\frac{a_{m}\left(y_{m}-d_{m}\right)}{x_{m-1}}=\frac{c_{m}\left(x_{m}-b_{m}\right)}{y_{m-1}}=\frac{a_{m} c_{m}}{x_{m-1} y_{m-1}-z_{m-1}} .
$$

The second and third of these four fractions are equal to the fourth when (and only when) $b_{m}$ and $d_{m}$ are defined in terms of $a_{m}, c_{m}, x_{m}, y_{m}, x_{m-1}, y_{m-1}$, $z_{m-1}$ by the formulæ

$$
b_{m}=x_{m}-\frac{a_{m} y_{m-1}}{x_{m-1} y_{m-1}-z_{m-1}}, \quad d_{m}=y_{m}-\frac{c_{m} x_{m-1}}{x_{m-1} y_{m-1}-z_{m-1}} .
$$

With these values of $b_{m}$ and $d_{m}$, the first fraction is equal to the fourth when (and only when) the product $a_{m} c_{m}$ is such that

$$
\frac{a_{m} c_{m} x_{m-1} y_{m-1}}{\left(x_{m-1} y_{m-1}-z_{m-1}\right)^{2}}-z_{m}=\frac{a_{m} c_{m}}{x_{m-1} y_{m-1}-z_{m-1}}
$$

i.e., when

$$
a_{m} c_{m}=z_{m}\left(x_{m-1} y_{m-1}-z_{m-1}\right)^{2} / z_{m-1} \text {. }
$$

We are now in a position to select the fourth sequence; we may, if we please, select the terms of the sequence $\left(a_{m}\right)$ arbitrarily, save that no member of it may be zero, and take it to be the fourth sequence. Then each member of the sequences $\left(a_{m}\right),\left(b_{m}\right),\left(c_{m}\right),\left(d_{m}\right)$ is readily expressible in terms of members of the sequences $\left(x_{m}\right),\left(y_{m}\right),\left(z_{m}\right),\left(a_{m}\right)$. Another possibility would, of course, be to take $\left(c_{m}\right)$ as the fourth sequence in place of $\left(a_{m}\right)$. It is, however, more satisfactory to regard the sequence $\left(a_{m}\right)$ as arbitrary and then to take as the fourth sequence the products of $a_{m}$ and convenient simple functions of $x_{m}$, $y_{m}, z_{m}$. Accordingly we write

$$
t_{m}=\frac{a_{m} z_{m-1}}{x_{m} y_{m-1}-z_{m-1}}, \quad(m=1,2, \ldots, n),
$$

and we can regard either the sequence $\left(t_{m}\right)$ or the sequence $\left(a_{m}\right)$ as arbitrary, whichever we prefer. We then have

$$
\begin{array}{lll}
a_{m}=t_{m}\left(x_{m-1} y_{m-1}-z_{m-1}\right) / z_{m-1}, & c_{m}=z_{m}\left(x_{m-1} y_{m-1}-z_{m-1}\right) / t_{m}, \\
b_{m}=x_{m}-y_{m-1} t_{m} / z_{m-1}, & d_{m}=y_{m}-x_{m-1} z_{m} / t_{m} ;
\end{array}
$$

and these values of $a_{m}, b_{m}, c_{m}, d_{m}$ satisfy all the conditions that we have enunciated.

To sum up our results, we see that the most general choice of $a_{m}, b_{m}, c_{m}, d_{m}$ which transforms the bilinear relation

$$
\left(\theta_{m}+x_{m}\right)\left(\phi_{m}+y_{m}\right)=z_{m}
$$

into the bilinear relation

$$
\left(\theta_{m-1}+x_{m-1}\right)\left(\phi_{m-1}+y_{m-1}\right)=z_{m-1}
$$

is the set of expressions just given. Moreover, these values of $a_{m}, b_{m}, c_{m}, d_{m}$ 
ensure that $\theta_{0}$ is expressible in terms of $\theta_{n}$ by means of the continued fraction

$$
\theta_{0}=\frac{a_{1} \mid}{\mid b_{1}}+\frac{a_{2} \mid}{\mid b_{2}}+\ldots \frac{a_{n} \mid}{\mid b_{n}+\theta_{n}}
$$

with a corresponding expression of $\phi_{0}$ in terms of $\phi_{n}$. The analysis requisite to establish my theorem is now complete. The result, stated in full, is as follows :

Theorem. Let $n$ be any positive integer, and let $\left(x_{m}\right),\left(y_{m}\right),\left(z_{m}\right)$ with $0 \leqslant m \leqslant n$ and $\left(t_{m}\right)$ with $1 \leqslant m \leqslant n$ be four arbitrary sequences. For $1 \leqslant m \leqslant n$ write

$$
\begin{array}{ll}
a_{m}=t_{m}\left(x_{m-1} y_{m-1}-z_{m-1}\right) / z_{m-1}, & c_{m}=z_{m}\left(x_{m-1} y_{m-1}-z_{m-1}\right) / t_{m}, \\
b_{m}=x_{m}-y_{m-1} t_{m} / z_{m-1}, & d_{m}=y_{m}-x_{m-1} z_{m} / t_{m} .
\end{array}
$$

Next let $\theta_{n}$ and $\phi_{n}$ be two variables connected by the relation

$$
\left(\theta_{n}+z_{n}\right)\left(\phi_{n}+y_{n}\right)=z_{n} .
$$

Then, in consequence of this relation, the product of the two following continued fractions, namely

$$
\left(x_{0}+\frac{a_{1} \mid}{\mid b_{1}}+\frac{a_{2} \mid}{\mid b_{2}}+\ldots+\frac{a_{n} \mid}{\mid b_{n}+\theta_{n}}\right) \times\left(y_{0}+\frac{c_{1} \mid}{\mid d_{1}}+\frac{c_{2} \mid}{\mid d_{2}}+\ldots+\frac{c_{n} \mid}{\mid d_{n}+\phi_{n}}\right),
$$

is, in general, equal to $z_{0}$.

3. The Transformation of Bauer and Muir as a Special Case of the Theorem of $\$ 2$

Apart from a trivial change of notation the BM theorem, as established by Perron, reads as follows:

The product of the two continued fractions

$$
\begin{aligned}
& \left(-\rho_{1} \alpha_{1}+\frac{\alpha_{1}\left(1+\rho_{1} \beta_{1}\right) \mid}{\mid \beta_{1}-\rho_{2} \alpha_{2}}+\ldots \frac{\alpha_{n}\left(1+\rho_{n} \beta_{n}\right) \mid}{\mid \beta_{n}-\rho_{n+1} \alpha_{n+1}}\right) \\
& \times\left(\beta_{1}+\frac{\alpha_{2}\left(1+\rho_{1} \beta_{1}\right) \mid}{\mid \frac{\beta_{2}-\rho_{1} \alpha_{2}}{\mid}}+\ldots+\frac{\alpha_{n}\left(1+\rho_{n-1} \beta_{n-1}\right) \mid}{\mid \frac{\beta_{n+1}\left(1+\rho_{n} \beta_{n}\right) \mid}{\beta_{n}-\rho_{n-1} \alpha_{n}}}+\frac{\frac{\alpha_{n+1}}{\mid \beta_{n+1}^{\prime}-\rho_{n} \alpha_{n+1}}}{\mid \beta_{0}}\right)
\end{aligned}
$$

(with $\beta_{n+1}^{\prime}$ equal to $-1 / \rho_{n+1}$ ) is equal to $\alpha_{1}$.

The change is the introduction of $\beta_{n+1}^{\prime}$; Perron wrote $\beta_{n+1}$ where we write $\beta_{n+1}^{\prime}$, so that we avoid imposing a restriction on $\beta_{n}$ which is not imposed on $\beta_{m}$ with $1 \leqslant m \leqslant n$. The object of the change is, of course, a slight gain in clarity.

To exhibit the BM theorem as a special case of my theorem, we assign to $x_{m}, y_{m}, z_{m}, t_{m}$ the following set of values:

$$
x_{m}=-\rho_{m+1} \alpha_{m+1}, \quad y_{m}=\beta_{m+1}, \quad z_{m}=\alpha_{m+1}, \quad t_{m}=-\alpha_{m}, \quad(0 \leqslant m \leqslant n),
$$

so that my theorem is specialised by the assumption that

$$
t_{m}=-z_{m-1}, \quad(1 \leqslant m \leqslant n) .
$$


We further specialise my theorem by taking $\theta_{n}$ to be zero, so that

$$
\phi_{n}=-y_{n}+\left(z_{n} / x_{n}\right) \text {. }
$$

The elements of my continued fraction are now given by the formulæ

$$
\begin{array}{ll}
a_{m}=\alpha_{m}\left(1+\rho_{m} \beta_{m}\right), & c_{m}=\alpha_{m+1}\left(1+\rho_{m} \beta_{m}\right), \\
b_{m}=\beta_{m}-\rho_{m+1} \alpha_{m+1}, & d_{m}=\beta_{m+1}-\rho_{m} \alpha_{m+1} .
\end{array}
$$

We also have

$$
\begin{aligned}
d_{n}+\phi_{n} & =\beta_{n+1}-\rho_{n} \alpha_{n+1}-\beta_{n+1}-\left(1 / \rho_{n+1}\right) \\
& =\beta_{n+1}^{\prime}-\rho_{n} \alpha_{n+1} .
\end{aligned}
$$

It is now clear that the consequence of specialising my theorem in this way is to yield precisely the BM theorem ; and it is arguable that this method of establishing the BM theorem is simpler and more straightforward than the method used by Bauer and Muir.

\section{An Apparent Generalisation of the Transformation of Bauer and Muir}

In deriving the BM theorem from my theorem in $\S 3$, we made specialisations of two different types, symbolised by the equations $t_{m}=-z_{m-1}$ and $\theta_{n}=0$. We now show how, by appropriate changes in notation, we can modify the BM theorem in such a way that it contains an arbitrary element $\theta_{n}$ at the end of the first of the two fractions and a corresponding element $\phi_{n}$ at the end of the second, thus partially bridging the gap which separates the BM theorem from my theorem.

We take the BM theorem in the form given in $\S 3$ and then, keeping the values of $\alpha_{m}$ and $\rho_{m}(1 \leqslant m \leqslant n+1)$ and of $\beta_{m}(1 \leqslant m \leqslant n)$ unaltered, we introduce additional elements, $\alpha_{n+2}, \rho_{n+2}, \beta_{n+1}^{\prime}\left(=-1 / \rho_{n+2}\right), \beta_{n+1}$. The BM theorem of order $n+\mathrm{l}$ (in place of $n$ ) with this complete set of elements asserts that

$$
\begin{aligned}
\left(-\rho_{1} \alpha_{1}+\frac{\alpha_{1}\left(1+\rho_{1} \beta_{1}\right) \mid}{\mid \beta_{1}-\rho_{2} \alpha_{2}}+\right. & \left.\ldots+\frac{\alpha_{n+1}\left(1+\rho_{n+1} \beta_{n+1}\right) \mid}{\mid \beta_{n+1}-\rho_{n+2} \alpha_{n+2}}\right) \\
& \times\left(\beta_{1}+\frac{\alpha_{2}\left(1+\rho_{1} \beta_{1}\right) \mid}{\mid \beta_{2}-\rho_{1} \alpha_{2}}+\ldots \frac{\alpha_{n+2}\left(1+\rho_{n+1} \beta_{n+1}\right) \mid}{\mid \beta_{n+2}^{\prime}-\rho_{n+1} \alpha_{n+2}}\right)=\alpha_{1} .
\end{aligned}
$$

We next define $\theta_{n}$ and $\phi_{n}$ by the formulæ

$$
\theta_{n}=\frac{\alpha_{n+1}\left(1+\rho_{n+1} \beta_{n+1}\right)}{\beta_{n+1}-\rho_{n+2} \alpha_{n+2}}, \quad \phi_{n}=\frac{\alpha_{n+1}\left(1+\rho_{n+1} \beta_{n+1}\right)}{\beta_{n+2}^{\prime}-\rho_{n+1} \alpha_{n+2}} .
$$

Since it is permissible to regard $\alpha_{n+2}$ as a variable, we may regard $\theta_{n}$ and $\phi_{n}$ as variables connected by the relation which is obtained by eliminating $\alpha_{n+2}$ from these two equations. By comparing the values of $\alpha_{n+2}$ in terms of $\theta_{n}$ and $\phi_{n}$ which are obtained by solving the equations for $\alpha_{n+2}$, we see that the relation may be written in the form

$$
\frac{\theta_{n} \beta_{n+1}-\alpha_{n+1}\left(1+\rho_{n+1} \beta_{n+1}\right)}{\theta_{n} \rho_{n+2}}=\frac{\phi_{n} \beta_{n+2}^{\prime}}{\phi_{n} \rho_{n+1}+\left(1+\rho_{n+1} \beta_{n+1}\right)},
$$

E.M.S. $-\mathrm{L} 2$ 
whence it follows that

$$
\left\{\theta_{n} \beta_{n+1}-\alpha_{n+1}\left(1+\rho_{n+1} \beta_{n+1}\right)\right\}\left\{\phi_{n} \rho_{n+1}+\left(1+\rho_{n+1}+\left(1+\rho_{n+1} \beta_{n+1}\right)\right\}+\theta_{n} \phi_{n}=0 .\right.
$$

When we discard the factor $1+\rho_{n+1} \beta_{n+1}$ (which we may assume not to vanish, since, if it did, we should have the relation of order $n$ only and not of order $n+1$ ), the relation becomes (as might have been expected)

$$
\left(\theta_{n}-\alpha_{n+1} \rho_{n+1}\right)\left(\phi_{n}+\beta_{n+1}\right)=\alpha_{n+1} .
$$

This relation has the consequence that the product of the continued fractions

$$
\begin{aligned}
\left(-\rho_{1} \alpha_{1}+\frac{\alpha_{1}\left(1+\rho_{1} \beta_{1}\right) \mid}{\mid \beta_{1}-\rho_{2} \alpha_{2}}+\ldots\right. & \left.+\frac{\alpha_{n}\left(1+\rho_{n} \beta_{n}\right) \mid}{\mid \beta_{n}-\rho_{n+1} \alpha_{n+1}}+\theta_{n}\right) \\
& \times\left(\beta_{1}+\frac{\alpha_{2}\left(1+\rho_{1} \beta_{1}\right) \mid}{\mid \beta_{2}-\rho_{1} \alpha_{2}}+\ldots+\frac{\alpha_{n+1}\left(1+\rho_{n} \beta_{n}\right) \mid}{\mid \beta_{n+1}-\rho_{n} \alpha_{n+1}}+\phi_{n}\right)
\end{aligned}
$$

is equal to $\alpha_{1}$.

When we write

$$
\rho_{m+1} \alpha_{m+1}=-x_{m}^{\prime}, \quad \beta_{m+1}=y_{m}^{\prime}, \quad \alpha_{m+1}=z_{m}^{\prime}, \quad(0 \leqslant m \leqslant n),
$$

we find that a consequence of the relation

$$
\left(\theta_{n}+x_{n}^{\prime}\right)\left(\phi_{n}+y_{n}^{\prime}\right)=z_{n}^{\prime}
$$

is that the product of the continued fractions

$$
\left(x_{0}^{\prime}+\frac{a_{1}^{\prime} \mid}{\mid b_{1}^{\prime}}+\frac{a_{2}^{\prime} \mid}{\mid b_{2}^{\prime}}+\ldots+\frac{a_{n}^{\prime} \mid}{\mid b_{n}^{\prime}+\theta_{n}}\right) \times\left(y_{0}^{\prime}+\frac{c_{1}^{\prime} \mid}{\mid d_{1}^{\prime}}+\frac{c_{2}^{\prime} \mid}{\mid d_{2}^{\prime}}+\ldots+\frac{c_{n}^{\prime} \mid}{\mid d_{n}^{\prime}+\phi_{n}}\right)
$$

is equal to $z_{0}$; in this result we have adopted the following abbreviations :

$$
\begin{array}{ll}
a_{m}^{\prime}=z_{m-1}^{\prime}-x_{m-1}^{\prime} y_{m-1}^{\prime}, & c_{m}^{\prime}=-z_{m}^{\prime}\left(x_{m-1}^{\prime} y_{m-1}^{\prime}-z_{m-1}^{\prime}\right) / z_{m-1}^{\prime}, \\
\dot{b}_{m}^{\prime}=x_{m}^{\prime}+y_{m-1}^{\prime}, & d_{m}^{\prime}=x_{m-1}^{\prime} z_{m}^{\prime} / z_{m-1}^{\prime}+y_{m}^{\prime} .
\end{array}
$$

In view of the definitions of the sequences $\left(x_{m}^{\prime}\right),\left(y_{m}^{\prime}\right),\left(z_{m}^{\prime}\right)$ in terms of the sequences $\left(\alpha_{m+1}^{\prime}\right),\left(\beta_{m+1}^{\prime}\right),\left(\rho_{m+1}^{\prime}\right)$, we may regard either the first three sequences or the second three as being given arbitrarily, whichever is more convenient.

This result is the most general and symmetrical version of the BM theorem, which is deducible either from the work of Bauer and Muir or from my theorem.

\section{The Derivation of the Theorem of $\S 2$ from the Theorem of Bauer and Muir}

We shall finally show how to remove one of the sequences from my theorem as enunciated in $\S 2$, and then to establish it with the help of the BM theorem, assuming, of course, that the BM theorem has already been proved by some means other than by an application of my theorem, e.g. by the methods used by Bauer and Muir. 


\section{A THEOREM ON CONTINUED FRACTIONS}

We take the two continued fractions introduced in $\$ 2$ and observe that, whatever be the arbitrary sequence $\left(u_{m}\right)$ with $0 \leqslant m \leqslant n$, elementary transformations of continued fractions yield the following results :

$$
\begin{aligned}
& x_{0}+\frac{a_{1} \mid}{\mid b_{1}}+\frac{a_{2} \mid}{\mid b_{2}}+\ldots+\frac{a_{n} \mid}{\mid b_{n}+\theta_{n}} \\
& \quad=\frac{1}{u_{0}}\left(u_{0} x_{0}+\frac{u_{0} u_{1} a_{1} \mid}{\mid u_{1} b_{1}}+\frac{u_{1} u_{2} a_{2} \mid}{\mid u_{2} b_{2}}+\ldots+\frac{u_{n-1} u_{n} a_{n} \mid}{\mid u_{n} b_{n}+u_{n} \theta_{n}}\right), \\
& y_{0}+\frac{c_{1} \mid}{\mid d_{1}}+\ldots+\frac{c_{n} \mid}{\mid d_{n}+\phi_{n}}=u_{0}\left(u_{0}^{-1} y_{0}+\frac{u_{0}^{-1} u_{1}^{-1} c_{1} \mid}{\mid u_{1}^{-1} d_{1}}+\ldots+\frac{u_{n-1}^{-1} u_{n}^{-1} c_{n} \mid}{\mid u_{n}^{-1} d_{n}+u_{n}^{-1} \phi_{n}}\right) .
\end{aligned}
$$

We now choose the sequence $\left(u_{m}\right)$ so that

$$
u_{m-1} u_{m}=-z_{m-1} / t_{m}, \quad(1 \leqslant m \leqslant n) ;
$$

this choice can be effected by taking $u_{0}$ arbitrary and

$$
\begin{gathered}
u_{2 m-1}=-\frac{t_{2} t_{4} \ldots t_{2 m-2} z_{0} z_{2} \ldots z_{2 m-2}}{t_{1} t_{3} \ldots t_{2 m-1} z_{1} z_{3} \ldots z_{2 m-3} u_{0}}, \quad\left(1 \leqslant m \leqslant \frac{1}{2} n+\frac{1}{2}\right), \\
u_{2 m}=\frac{t_{1} t_{3} \ldots t_{2 m-1} z_{1} z_{3} \ldots z_{2 m-1} u_{0}}{t_{2} t_{4} \ldots t_{2 m} z_{0} z_{2} \ldots z_{2 m-2}}, \quad\left(1 \leqslant m \leqslant \frac{1}{2} n\right)
\end{gathered}
$$

with $m$ running through integral values only in both cases.

Now that the sequence $\left(u_{m}\right)$ has been constructed, we define two new sequences $\left(x_{m}^{\prime}\right)$ and $\left(y_{m}^{\prime}\right)$ to replace the sequences $\left(x_{m}\right)$ and $\left(y_{m}\right)$; we write

$$
\dot{x_{m}}=u_{m} x_{m}, \quad y_{m}^{\prime}=u_{m}^{-1} u^{-1} y_{m}, \quad(0 \leqslant m \leqslant n),
$$

and then we define $a_{m}^{\prime}, b_{m}^{\prime}, c_{m}^{\prime}, d_{m}^{\prime}(1 \leqslant m \leqslant n)$ by the formulæ

$$
a_{m}^{\prime}=u_{m-1} u_{m} a_{m}, b_{m}^{\prime}=u_{m} b_{m}, c_{m}^{\prime}=u_{m-1}^{-1} u_{m}^{-1} c_{m}, d_{m}^{\prime}=u_{m}^{-1} d_{m},
$$

so that we have

$$
\begin{array}{ll}
a_{m}^{\prime}=z_{m-1}-x_{m-1}^{\prime} y_{m-1}^{\prime}, & c_{m}^{\prime}=-z_{m}\left(x_{m-1}^{\prime} y_{m-1}^{\prime}-z_{m-1}\right) / z_{m-1}, \\
b_{m}^{\prime}=x_{m}^{\prime}+y_{m-1}^{\prime}, & d_{m}^{\prime}=x_{m-1}^{\prime} z_{m} / z_{m-1}+y_{m}^{\prime} .
\end{array}
$$

Consequently $a_{m}^{\prime}, b_{m}^{\prime}, c_{m}^{\prime}, d_{m}^{\prime}$ have the values assigned to them in $\S 4$, it being supposed that $z_{m}^{\prime}$ and $z_{m}$ are identical.

We now have

$$
\begin{aligned}
& x_{0}+\frac{a_{1} \mid}{\mid b_{1}}+\frac{a_{2} \mid}{\mid b_{2}}+\ldots+\frac{a_{n} \mid}{\mid b_{n}+\theta_{n}}=\frac{1}{u_{0}}\left(x_{0}^{\prime}+\frac{a_{1}^{\prime} \mid}{\mid b_{1}^{\prime}}+\frac{a_{2}^{\prime} \mid}{\mid b_{2}^{\prime}}+\ldots+\frac{a_{n}^{\prime}}{\mid b_{n}^{\prime}+u_{n} \theta_{n}}\right) \\
& y_{0}+\frac{c_{1} \mid}{\mid d_{1}}+\frac{c_{2} \mid}{\mid d_{2}}+\ldots+\frac{c_{n} \mid}{\mid d_{n}+\phi_{n}}=u_{0}\left(y_{0}^{\prime}+\frac{c_{1}^{\prime} \mid}{\mid d_{1}^{\prime}}+\frac{c_{2}^{\prime} \mid}{\mid d_{2}^{\prime}}+\ldots+\frac{c_{n}^{\prime}}{\mid d_{n}^{\prime}+u_{n}^{-1} \phi_{n}}\right)
\end{aligned}
$$

and, since $\theta_{n}$ and $\phi_{n}$ are connected by the relation

$$
\left(u_{n} \theta_{n}+x_{n}^{\prime}\right)\left(u_{n}^{-1} \phi_{n}+y_{n}^{\prime}\right)=z_{n}
$$


it follows from $\$ 4$ that the product of the continued fractions on the right is equal to $z_{0}$.

Hence the product of the continued fractions on the left is also equal to $z_{0}$, with $\theta_{n}$ and $\phi_{n}$ satisfying the relation

$$
\left(\theta_{n}+x_{n}\right)\left(\phi_{n}+y_{n}\right)=z_{n}
$$

and this is precisely the result which I established otherwise in $\S 2$.

Consequently the BM theorem and my theorem of $\S 2$ are equivalent in the sense that each is deducible from the other by simple and straightforward analysis.

It would seem that there is not much more to be done in the way of elucidating the BM theorem, if indeed I have not now said the last word on the subject.

\section{BIBLIOGRAPHY}

(1) G. Bauer, Abh. der k.b. Akad. der Wiss. zu München, 11 (1872), II.

(2) T. Muir, Phil. Mag. (5), 3 (1877), 137-138, 360-366.

(3) O. Perron, Die Lehre von den Kettenbrüchen (1912, 1929, 1954-7); see especially $\S \S 47$ and 42 of the edition of 1929.

46 WARWICK NEW ROAD

Leamington, WaRWICKSHIRE 